\title{
An observational study of perioperative nutrition and postoperative outcomes in patients undergoing laparotomy at Queen Elizabeth Central Hospital in Blantyre, Malawi
}

\author{
Kondwani G.H. Katundu ${ }^{1}$, Timothy W. Mutafya ${ }^{2}$, Noel C. Lozani ${ }^{2}$, \\ Patrick M. Nyirongo ${ }^{2}$, Molly E. Uebele ${ }^{3}$, \\ 1. Division of Physiology, College of Medicine, University of Malawi, Blantyre, Malawi \\ 2. College of Medicine, University of Malawi, Blantyre, Malawi \\ 3. College of Medicine, University of Malawi, Blantyre, Malawi
}

Date Received: 16 May 2017

Revision Received: 19 July 2017

Date Accepted: 31 August 2017

Correspondence:

Kondwani G.H. Katundu

(kkatundu@medcol.mw)

https://dx.doi.org/10.4314/mmj.v30i2.5

\section{Background}

Abstract

Nutritional status in patients undergoing surgery can influence their immune function, tissue repair and, hence, clinical outcomes. This study aimed to assess the perioperative nutrition and postoperative outcome of patients undergoing laparotomy at a tertiary hospital in Malawi.

\section{Methods}

A total of 25 patients were included in this prospective, observational study. The Subjective Global Assessment was used to classify each patient according to nutritional status. Handgrip strength was measured for each patient preoperatively and at day 3 postoperatively. Anthropometric measurements were also done. Protein and energy requirements for each participant were estimated and compared to the quantities provided by the hospital diet. Patients were followed up until discharge and outcome variables which included length of hospital stay and wound dehiscence or infectious complications were recorded.

\section{Results}

Of the study participants, $20 \%$ were well-nourished, $52 \%$ were moderately malnourished and 28\% were severely malnourished. The median handgrip strength decreased at day 3 postoperatively from the preoperative handgrip strength. Well-nourished patients had higher handgrip strength than malnourished patients both preoperatively and postoperatively. Total energy and protein provided by the hospital diet were significantly lower than the estimated requirements for the patients. Severely malnourished patients had increased median length of hospital stay and increased rate of postoperative complications. Preoperative and postoperative day 3 handgrip strength correlated negatively with the number of postoperative complications and length of hospital stay.

\section{Conclusion}

This study showed high rates of malnutrition and inadequate in-hospital nutritional support which were associated with poor clinical outcomes, especially in severely malnourished patients. Proper nutritional assessment and provision of adequate nutritional support should be reinforced in surgical patients to promote favourable clinical outcomes postoperatively. Further studies with larger sample sizes in other patient populations and hospitals in Malawi are required in this area.

\section{Introduction}

Malnutrition remains a challenge and is highly prevalent among in-patients ${ }^{1,2}$. Globally, current data suggest that 19 to $59 \%$ of hospitalised adults have malnutrition, with the higher range in low-income and middle-income countries ${ }^{3-10}$. Malnutrition is of special importance for the surgical patient due to its influence on postoperative patient outcomes. The association between preoperative weight loss and surgical mortality was first documented in 1936 by Studley ${ }^{11}$. Since then, considerable evidence has shown that preoperative malnutrition is associated with postoperative complications, including infection, delayed wound healing, and increased length of hospital stay ${ }^{12}$. Furthermore, several studies have shown that treatment of severe malnutrition before surgery improves postoperative clinical outcomes ${ }^{13-15}$.

Malnutrition refers to a state of inadequate or excess nutrition, with or without the presence of inflammation, which leads to changes in body composition and impaired physiologic function $^{16}$. An individual who presents for surgical evaluation may have comorbidities that predispose to chronic diseaserelated malnutrition, such as HIV, organ failure, cancer, metabolic syndrome, and many other conditions characterised by persistent moderate inflammation ${ }^{17}$. Preoperative malnutrition could also result from simple starvation-related malnutrition, defined as chronic undernutrition in the absence of an inflammatory condition. Regardless of preoperative nutrition status, all patients are at an increased risk of acute disease-related malnutrition after surgery ${ }^{17}$. Any physiologic injury stimulates a metabolic stress response mediated by inflammatory cytokines and catecholamines. Collectively, these increase energy expenditure and muscle proteolysis in proportion to the severity of the injury ${ }^{18}$. Coupling this with decreased or restricted nutrient intake and decreased mobility postoperatively leads to rapid deterioration of lean body mass and function ${ }^{19}$. Thus, even a well-nourished or 
over-nourished patient who undergoes a major surgery can 28 participants with HIV infection and confirmed cancer develop severe acute disease-related malnutrition within as ittle as 10 days if not fed appropriately

Evidence-based recommendations are available to guide nutritional care in the perioperative period. Preoperative nutrition therapy recommendations are dependent on the be screened for malnutrition risk prior to surgery and evaluated by a dietitian if identified as high risk. If the patient is diagnosed with severe malnutrition and the patient is diagnosed with severe malnutrition and the
surgical intervention is not emergent, the surgery should be postponed 7 to 10 days while the patient is given appropriate
nutritional support ${ }^{20,21}$.

On the day of surgery, theEuropean Society of Anesthesiology recommends that patients be allowed to eat solid food up to 6 hours before surgery ${ }^{22}$. Intake of clear liquids is encourage should take nothing by mouth. The Enhanced Recovery After Surgery (ERAS) protocol, which has repeatedly shown significant benefits for patients undergoing gastrointestinal surgery includes preoperative carbohydrate loading with clear liquid beverage to possibly reduce postoperative insulin resistance, nausea, and vomiting ${ }^{22}$.

After surgery, most patients can resume a normal diet. There is no evidence that gastric decompression or fasting after surgery is beneficial ${ }^{23}$. Rather, enteral feeding or a metabolic stress response, surgical patients have increased protein and kilocalorie requirements after surgery 18 . At les $60^{\circ}$ of protein requirements should be met within 7 to 10 days to prevent complications of malnutrition ${ }^{20,24}$. Thus, the European Society of Enteral and Parenteral Nuthus, guidelines state that all patients should be fed by mouth soon as possible, preferably within 24 hours, or initiated on enteral tube feeding if not anticipated to eat orally in 7 days $\mathrm{s}^{25}$. In cases of intestinal failure, parenteral nutrition should be initiated as soon as possible for a malnourished patient, or nutrition does not seem feasible in the following 3-7 day $\mathrm{s}^{262}$. Curcenty, there is pacity of data on qualing Currently, there is paucity of data on the quality of nutrition care services offered to surgical patients in Malawi, against of data renceding patients in Malawi. Therefore, this prospective observation study was aimed at assessing the preoperative nutrition status and the currently available nutrion interventions for alot surgical patients undergoing laparotomy at a tertiary referra hospital in Mawi. The nutrition provided to patients by the hospital was evaluated for adequacy of protein and kilocalo content, as suroical patients require increased intake of the nutrients to mitigate the catabolic effect of metabolic stress. The influence of these factors on postoperative outcomes was also investigated.

\section{Methods}

\section{Study population}

This prospective observational study was conducted at Queen Elizabeth Central Hospital (QECH) in Blantyre,
Malawi, and enrollment was done between July and September 2016. Adult patients admitted to the male and female surgical wards and scheduled for laparotomy were of the main drivers. Because immunosuppression is one yagnosis were excluded from the study. Participants aged 65 years and above were also excluded. Using an alpha error of \% and power of $80 \%$, the sample size was calculated at 25 patients, to detect at least $20 \%$ prevalence of malnutrition in the study population. The study protocol was approved


(Written informed consent was sough and provided by all the participants before their inclusion 作 (antion provided, study codes paridual participants. Data that could identify the individual to

Nutrition assessment

The Subjective Global Assessment (SGA) was used to assess and classify each patient's nutritional status as severe SGA SGA ias B), or well nounished (SGA Class $A$ ). The tool that uses clinical history and physical exam nutrition system based on the clinician's subjective assessment (mil moderate, or severe) of the patient's reported dietary intake, weight changes, gastrointestinal symptoms and function capacity along with the degree of metabolic stress in their dingnosis, and a physical examination. A registered dieti. trined medical students who were part of the study tean ow to use the SGA method. capacity. Height and weight were measured preoperatively. (BMI). Popometics were used to calculate body mass index (BMI). Postoperative protein and energy requirements for each participant were also estimated. Energy (kilocalorie) needs requirements were dosed between 1.2-1.5 grams $/ \mathrm{kg}$ requirements were determined by an experienced registered dietitian, according to the severity of the surgery and other

\section{Outcome monitoring}

Patients were followed prospectively until discharge Outcome variables including length of hospital stay and development of wound dehiscence or infectious complications were recorded. An infectious complication was considered when there was persistent fever (temperature $\left.338^{\circ} \mathrm{C}\right) 72$ hours after surgery. Lastly, handgrip strength was measured in all patients on day 3 postoperative.

Menu analysis

The recipes for food provided to patients on a standard diet from the hospital kitchen were collected. The hospita menu was referenced and cross-checked with kitchen staff. estimated the average portion sizes by observing the mea service. All the food served to one patient in one day was then analysed for protein and kilocalorie content using the Tanzania Food Composition Table ${ }^{31}$. Since the menu is generally the same day to day, only one day's menu was analysed. (and not names) were used on the data collection sheets for

Handgrip dynamometry was used to assess functiona with an individualised stress factor of 1.2 or 1.3. Protein Portion sizes are not standardised. Therefore, the researcher

\section{Malawi Medical Journal 30 (2): 79-85 June 2018}

\section{Statistical analysis}

Statistical analysis of the data was done using Stata versio 2 (StataCorp, USA) and GraphPad Prizm version 7.01 assessed using the Shapiro-Wilks test. Descriptive data was were expressed as median (IQR) for the contre statistics The Wilcoxon rank-sum test (or Mann-Whitney U-test) was used to test the equality of two medians, to investigate differences in the mesured variables. To test for differences between medians of the analysed variables at different points in time, the Wilcoxon paired rank-sum test and the Friedman test (K-related samples) were used. To measure the associations between the variables, Spearman's test of association was used. A p-value of $<0.05$ was considered statistically significant.

\section{Results}

A total of 34 patients were screened and 25 patients who fit the study criteria were included (see Figure 1). The characteristics of the study population are given in Table 1. The sample was well distributed between males and females. The major indication for laparotomy in the study sample was prostatectomy due to benign prostatic hypertrophy and intra-abdominal tumours that had not yet been diagnosed by histopathology. With regards to post-operative complications, $72 \%$ of the study participants either developed incisionwound infection or dehiscence. No deaths were recorded from the participants during the study period. According to the BMI classification of nutritional status, most of the patients $(76 \%)$ had normal weight, only a few were overweight

Screened patients scheduled for laparotomy over the study period

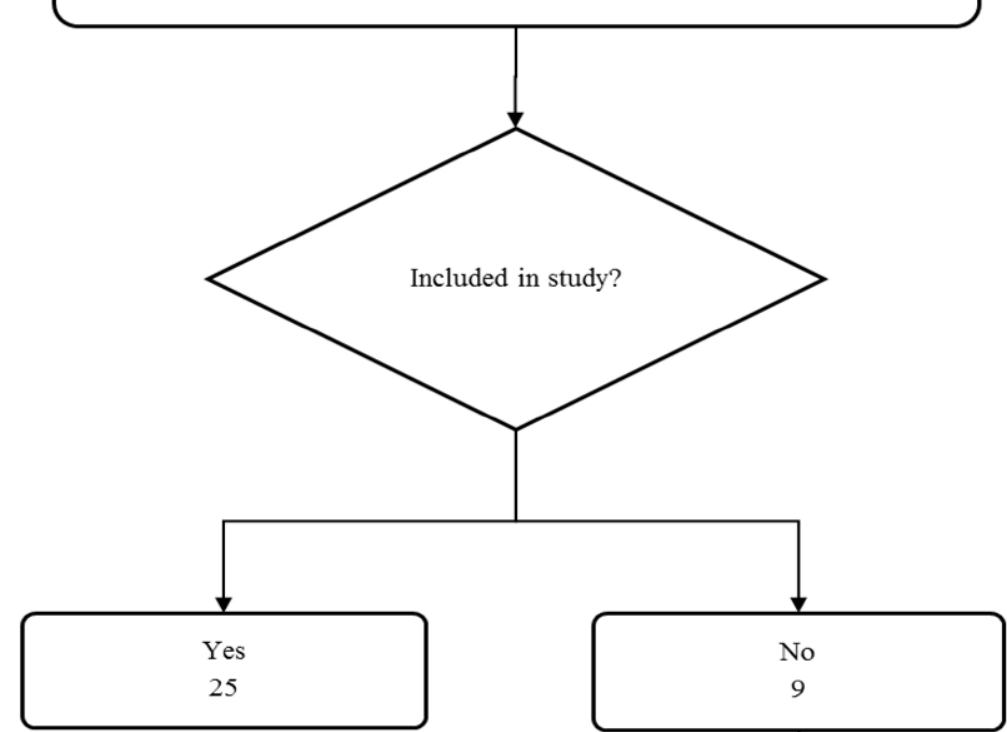

Perioperative nutrition and postoperative outcomes 81

Table 1. Characteristics of the study population

\begin{tabular}{|l|c|}
\hline $\begin{array}{l}\text { Gender, } \mathrm{n}(\%) \\
\text { Males }\end{array}$ & $13(52 \%)$ \\
Females & $12(48 \%)$ \\
\hline Age [years] median (lQR) & $38(28.5-49)$ \\
\hline Indication for laparotomy $\mathrm{n}(\%)$ & \\
Prostatectomy & $11(44 \%)$ \\
Bowel obstruction & $4(16 \%)$ \\
Intra-abdominal tumours & $8(32 \%)$ \\
Exploratory laparotomy & $2(8 \%)$ \\
& \\
\hline
\end{tabular}

$(6 \%)$ and none was underweight or obese (see Table 1 . Nevertheless, using the SGA classification of nutritional status, only $20 \%$ of the participants were categorised as well-nourished. The remaining $80 \%$ were either moderately malnourished $(52 \%)$ or severely malnourished $(28 \%)$.

Handgrip strength was assessed in this study to measure the functional capacity of the patients both preoperatively and day 3 postoperatively. Figure 2 shows a comparison of the handgrip strength between the two-time points. Notably, the median handgrip strength for the entire study group was $22.3 \mathrm{~kg}(20-33)$ preoperatively but decreased significantly to $18.4 \mathrm{~kg}$ $(14-27)$ postoperatively $(\mathrm{p}<0.0001)$. Handgrip strength was then analysed per SGA categories (Figure 3). Notably, the handgrip strength was associated with SGA class, where class A had a higher median handgrip strength than classes $B$ and C both preoperatively (class A $33 \mathrm{~kg}$ (25.25 - 40.4), class B $25.55 \mathrm{~kg}(21.85-34)$, class C $20 \mathrm{~kg}(16-22))(\mathrm{p}<0.0001)$ and
postoperatively (class A $27 \mathrm{~kg}(16.5-39)$, class B $20 \mathrm{~kg}(17.25-27.75)$, class C $14 \mathrm{~kg}$ $(12-15))(\mathrm{p}=0.0004)$.

The daily energy and protein requirement for each patient was calculated, accounting for each patient was calculated, accounting
for both the basal physiological needs and the stress associated with surgery. The typical diet provided by the hospital was porridge with some sugar in the morning and nsima (thick maize porridge) with beans, cabbage or both for lunch and supper.

Reasons for exclusion

Reasons for exclusion
HIV infected - 4
Confirmed diagnosis of Cancer - 1 Loss to follow-up - 2
No consent provided - 2 of the total energy and protein provided tho 5 dhe diet was calculated. Figures and 5 depict comparisons between the otal energy requirements and what was arovinst what was provided Bequirements and protein (31.54) provided by the 


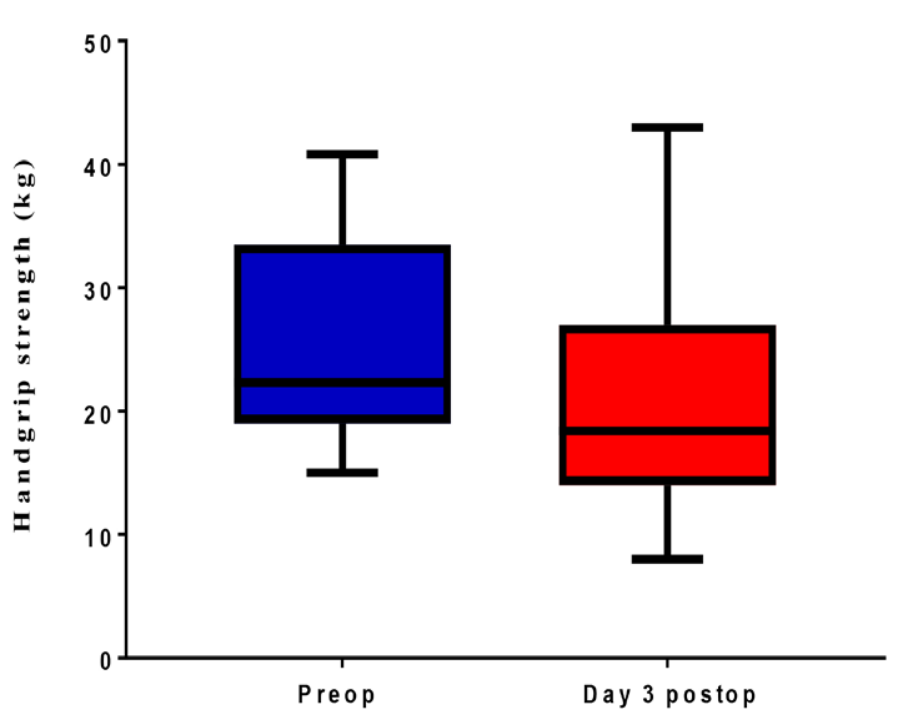

Time of handgrip strength assessm en

Figure 2. Box plot of handgrip strength of the dominant hand preoperatively and at day 3 postoperatively. The median handgrip preoperative handgrip strength (Wilcoxon paired rank-sum test <0.0001).
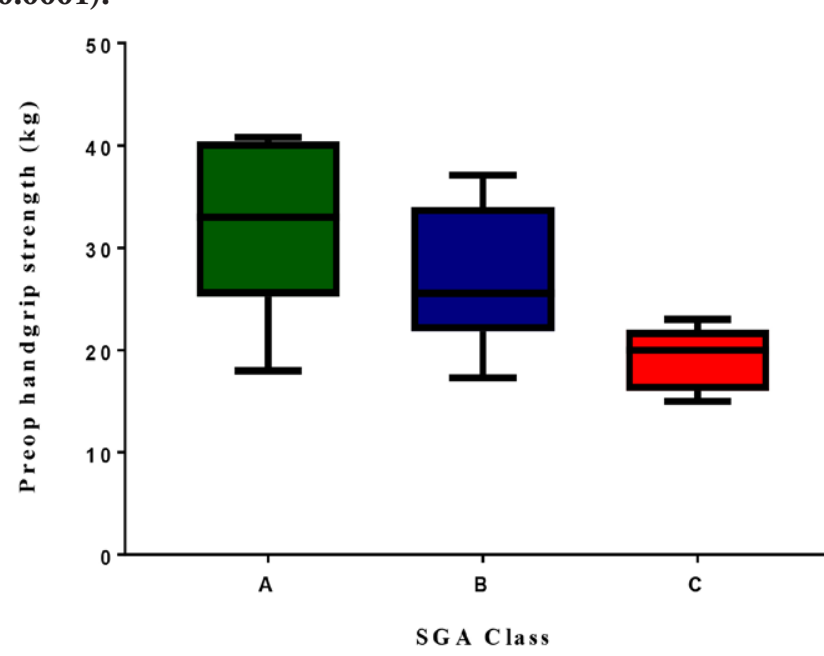

A.

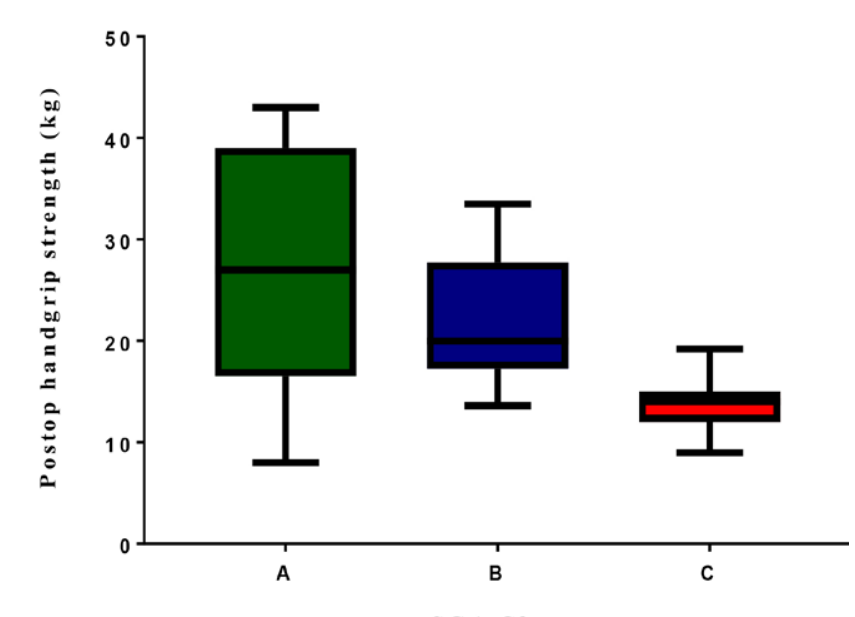

SGA Clas, hospital diet were statistically significantly lower than the estimated requirements (1564 kcal (1488 - 1809 and 72 $(60.52$ - 81) respectively) $(p<0.0001)$. According to the calculated estimates, none of the patients met their energy and protein requirements by the provided hospital diet alone. It should be noted, however, that none of the patients was provided with enteral or parenteral nutrition. Also, these calculations were based on a patient who solely depended on the nutritional support provided by the hospital, and hence no food items provided from other sources were included in the energy and protein analysis of this study. The proportion of postoperative complications in the study group was $72 \%$. Of all these complications, $59 \%$ were observed in patients who were in SGA class C, while those of class B contributed $31 \%$ and only $10 \%$ SGA class $\Lambda$. The media length of hospital stay was 5 days $(4-7)$. However, severely malnourished patients (SGA class $C$ ) had a median length of hospital stay of 8 days (7 - 9). Well-nourished patients (SGA class A) had a median length of stay of 4 days $(4-4)$.

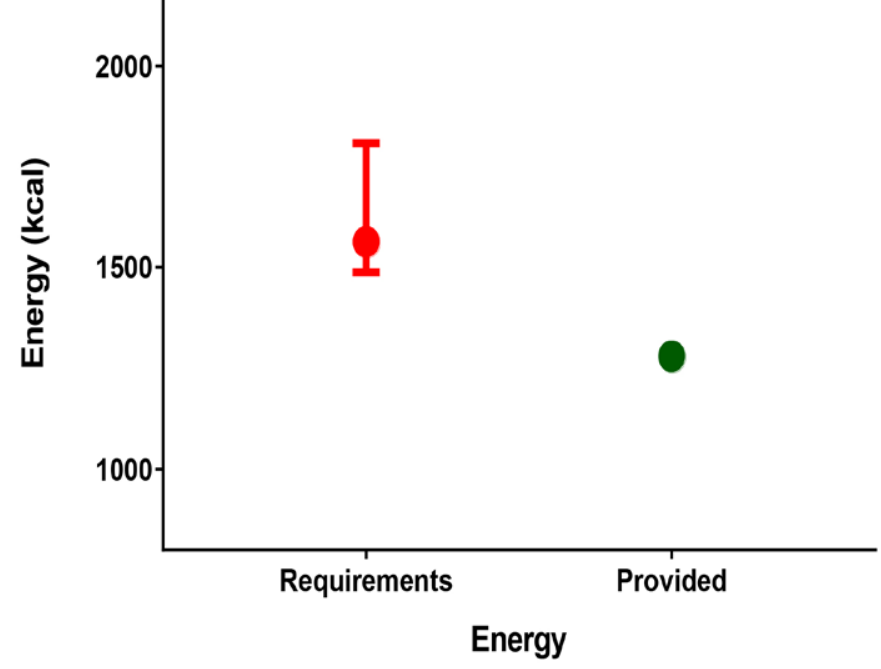

Figure 4. Comparison between the median (IQR) total energy requirements for the patients and the actual provision from the
hospital diet. Total energy provided was significantly lower than hospital diet. Total energy provided was significantly lower than the median energy requirement for patients (Wilcoxon rank-sum

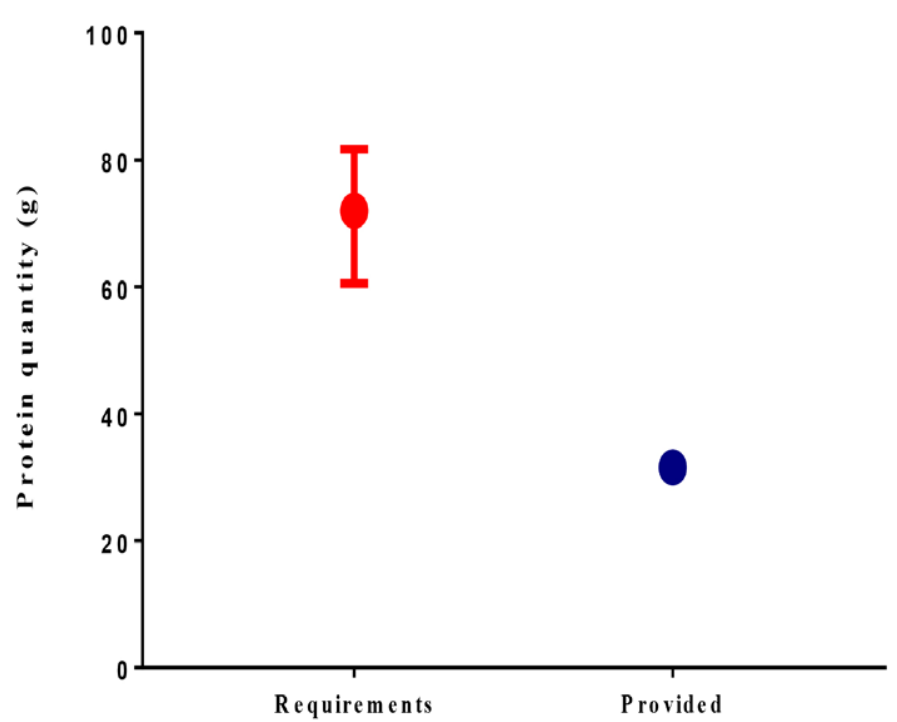

Protein

(A) and Handgrip strength among the SGA classes preoperatively (A) and at day 3 postoperatively (B). Lower SGA classes B and C had decreased handgrip strength compared to SGA class A using
Friedman test, both preoperatively $(\mathrm{p}<0.0001)$ and at day 3
postoperatively $(\mathrm{p}=\mathbf{0 . 0 0 0 4})$.
Fequire 5. Comparison between the median (IQR) protein
requirents for the patients against provision from the hospitas diet. Total protein provided was significantly lower than the medi protein requirement for patients using the Wilcoxon rank-sum test
$(\mathbf{p}<\mathbf{0 . 0 0 0 1})$.
Those who were mildly or moderately malnourished (class practical and cost-effective tool for Malawian hospitals ${ }^{38}$. B) had a median length of stay of 5 days $(4-5)(p<0.0001)$. Our finding that the severity of preoperative malnutrition Association between handgrip strength and outcome according to SGA class correlated with postoperative 3 postoperative hassed. Notably, both preoperative and day complications and length of hospital stay is consistent with negatively with the number of postoperative complications the literature ${ }^{5-7,12,39}$. Poor SGA class has also been associated and length of hospital stay (see Table 2). $\quad$ attention to the critical importance of nutritional assessment $\begin{array}{ll}\text { Table 2. Association between handgrip strength and outcome variables } & \text { by a trained professional } \\ \text { prior to surgery. Currently, }\end{array}$ handgrip strength v. complications handgrip strength $\mathbf{v}$. hospital stay to our knowledge, there is no Sormal nutritional screening $\begin{array}{ll}\text { Study time Spearman's rho } p \text {-value Spearman's rho } p \text {-value } & \text { or assessment performed in } \\ \text { adult inpatients at QECH. } & \text { Consequently, patients who }\end{array}$

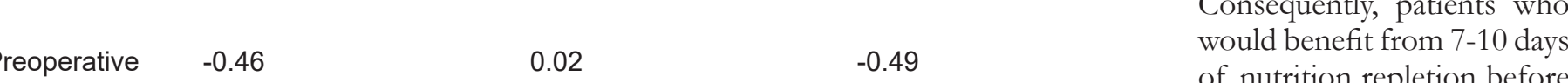
of nutrition repletion before

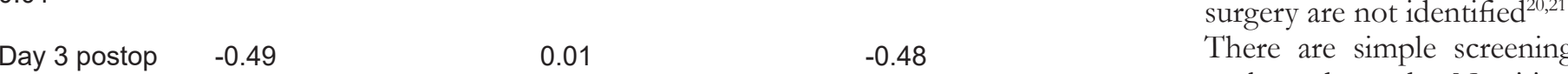
ools such as the Nutrition Risk Score 2002 (NRS 2002),

\section{Discussion}

Assesment (ANA) 2002 (NRS 2002)

Mini Nutrition Assessment (MNA) and the Malnutrition
Universal Screening Tool (MUST) that could be implemented To our knowledge, this is the first study to assess the to identify patients at high risk of malnutrition for potential nutritional status, nutritional interventions and outcomes preoperative nutrition therapy ${ }^{40}$.

in a subset of surgical patients undergoing laparotomy at a In this study, handgrip strength was significantly lower at that $80 \%$ of the patients had some degree of malnutrition day 3 postoperatively than that at preoperative assessment. with $28 \%$ severely malnourished. Considering that at least This indication of a decline in functional capacity due $39 \%$ of Malawins are food insecure and that patients at to loss of lean body mass ${ }^{19}$. Major surgery elicits metabolic QECH re referred from district hospitals that serve rutal responses associated with increased muscle proteolysis and communities, this result is not very surprising32 Other increased energy expenditure that puts patients at risk of acute disease-related malnutrition, where even an excess of lower rates of in-hospital adult malnutrition. Uanda and adipose tissue will not prevent catabolism of lean tissue ${ }^{17,18}$ Burundi reported rates of $47 \%$ and $59 \%$, respectively $3^{3,34}$. This catabolic state cannot be reversed via protein and However, low-income regions within Brazil found $78 \%$ of energy intake; however, adequate provision slows lean adult inpatients, of which more than $50 \%$ were suroical body mass wasting, mitigates the inflammatory response, patients, were malnourished ${ }^{8}$. Multiple upper-middle income and, if provided enterally, maintains the integrity of the countries have found about $50 \%$ of adult inpatients as gastrointestinal tract as a major immune organ ${ }^{25}$. Handgrip malnourished ${ }^{3,9}$ Even in high-income settinos, for example strength correlates highly with nutrition status in hospitalised Australia and the United States of America, the rate of patients, as is replicated in the present study. Moreover, this malnutrition in surgical inpatients may be as high as $48 \% .5,6$ tool objectively captures a change in nutrition status faster The higher rate of malnutrition in the current Malawion than any anthropometric or laboratory marker ${ }^{41}$. The present study, therefore, is likely due to the coupled effect of the study showed that handgrip strength both preoperatively disease processes and inadequate nourishment owing to and postoperatively correlated negatively with the length of food insecurity, which increases the risk of malnutrition in hospital stay and the rate of complications postoperatively. the population. These results indicate that in-hospital decline in nutritional Nutrition in this study was assessed using the SGA status was associated with increased risk of complication These findings, therefore, call to the importance of both che optimal nutritional status of patients before surger for the postoperatively. the SGeal of nutrition therapy after surgery is to ensure the andernourished (BMI <18.5). This confirms the limitation preservation of lean body mass and immune function, which of BMI as a less sensitive tool for assessing malnutrition is essential for healing in these patients $s^{42,43}$. For a patient who in acute and complicated clinical conditions which obscure already exhibits severe muscle wasting, it is imperative tha indernutrition 35.36 . BMI is a useful measure in the general oral, enteral, or parenteral nutrition support be initiated a population, where it correlates with mortality although the soon as possible after surgery ${ }^{26}$. Postoperatively, the usua tradition cutoffs for underweight $\left(<18.5 \mathrm{~kg} / \mathrm{m}^{2}\right)$, overweight practice at QECH is to start the patient on a liquid diet only $\left(>25-29.9 \mathrm{~kg} / \mathrm{m}^{2}\right)$, and obesity $\left(>30 \mathrm{~kg} / \mathrm{m}^{2}\right)$ are derived after 24 hours and in the presence of bowel sounds. The from data in predominantly Caucasian populations ${ }^{37}$. In an diet is then gradually advanced to a regular solid diet. In acutely ill individual, when metabolic changes and fluid shifts special cases, clinicians prescribe a high protein diet (HPD), occur rapidly, BMI is not useful because weight changes are which might include an egg and milk. However, HPD confounded by fluid status ${ }^{38}$. The use of SGA is, therefore, rarely feasible for the kitchen to provide due to budgetar preferable to BMI in such clinical settings, Since SGA does constrains. Otherwise, there are no speciallsed nutritiona not rely on laboratory or anthropometric measures, it is a interventions avallable. The analysis of the standard hospit 
(284.2kcal) and protein quantity $(40.46 \mathrm{~g})$. Such a deficient and had increased length of hospital stay compared to diet cannot ameliorate lean body mass wasting. It is also well-nourished patients. The standard diet provided at the inadequate for tissue repair and several immune functions hospital for the patients was deficient in both energy and In an adult. According to ESPEN guidelines, four patients protein. Improved nutrition care may improve outcomes

$(16 \%)$ in the current study had indications for postoperative in surgical laparotomy patients. Further studies, with larger
parenteral nutrition, which could not be provided ${ }^{26}$. The sample sizes in other patient populations and hospitals in parenteral nutrition, which could not be provided ${ }^{26}$. The overall decline in handgrip strength and high rate of another indication of inadequate postoperative nutrition support. The resulting unmitigated muscle catabolism has consequences. Literature has shown that a $10 \%$ loss of lean mass is associated with impaired immunity and skin integrity, and that loss of $40-45 \%$ of lean body mass is incompatible with life ${ }^{42}$.

Appropriate nutritional management is an effective intervention. A recent meta-analysis of randomised controlled trials showed that nutrition interventions in malnourished patients reduced the risk of postoperative infectious complications by $42 \%$ and noninfectious complications by $26 \%{ }^{15}$. The length of hospital stay was also reduced by 2.6 days $^{15}$. Appropriate nutritional interventions are also cost-effective. Studies indicate that, on average, malnourished patients (SGA class B and C) have 31$38 \%$ higher hospital costs than well-nourished patients. 4 Nutritional interventions would, therefore, reduce morbidity, mortality and the associated hospital costs.

Given the findings of this study, nutrition interventions have the potential to catalyse significant improvement to healt care delivery in Malawi. Availability of registered clinic dietitians to assess and manage an individualised nutritio care plan is critical. Currently, there are no dietitians in Malawian government hospitals. However, a program to train dietitians is presently training its first cohort. Availability of adequate nutrients, in the form of food, supplements, enteral formula, and parenteral solutions is also essential. This study identifies the need for hospitals in Malawi to integrate nutritional management as an essential part of health service
delivery in adult patients, especially in conditions where the evidence points to its efficacy in improving outcomes.

\section{Study Limitations}

This study is limited by its design as an observational study, and, as such, can only report associations. Interpretation of the results of this study should be made in light of its design. The calculations of energy and protein content from the diet were based on a patient who solely depended on the nutritional support provided by the hospital, and hence no food items provided from other sources were included. Further to this, the calculations were based on what was provided by the hospital, and not on the actual intake. Considering that some patients may not have taken all the food provided, actual intake may have been lower than the provision. Another limitation of the study is its sample size of 25 , and even though this was adequate to provide baseline data on the topic, subgroup analyses may not be feasible. Lastly, due to the limited time frame for this study, we were unable to follow up patients after discharge to monito mortality or rate of readmission.

\section{Conclusions}

This observational study has shown that the prevalence of malnutrition in surgical patients undergoing laparotomy at malnutrition experienced more postoperative complications Malawi, are required in this area.

\section{Acknowledgements}

We acknowledge the office of the hospital director at Queen Elizabeth Central Hospital (QECH), for authorising the conduct of this study. We are also grateful to the head of Surgery Department at the hospital and staff members of period. Support from Doris Cement Nanga of the Faculty of Agriculture and Natural Resources, regarding food

\section{References}

1. Pathirana AK, Lokunarangoda N, Ranathunga I, Santharaj WS Ekanayake R, Jayawardena R: Prevalence of hospital malnutrition Springerplus 2014, 3:412-1801-3-412.

2. Souza TT, Sturion CJ, Faintuch J: Is the skeleton still in the hospital closet? A review of hospital malnutrition
aspects. Clin Nutr 2015, 34(6):1088-1092.

3. Correia MI, Campos AC, ELAN Cooperative Study: Prevalence of hospital malnutrition in Latin America: the multicenter ELAN study Nutrition 2003, 19(10):823-825.

4. Curtis LJ, Bernier P, Jeejeebhoy K, Allard J, Duerksen D, Gramlich L, Laporte M, Keller HH: Costs of hospital malnutrition. Clin Nutr 2017, 36(5):1391-1396

5. Garth AK, Newsome CM, Simmance N, Crowe TC: Nutritiona status, nutrition practices and post-operative complications in patien
with gastrointestinal cancer J Hum Nutr Diet 2010, 23(4):393-401.

6. Mosquera C, Koutlas NJ, Edwards KC, Strickland A, Vohra NA Zervos EE, Fitzgerald TL: Impact of malnutrition on gastrointestina surgical patients. J Surg Res 2016, 205(1):95-101.

7. Thomas MN, Kufeldt J, Kisser U, Hornung HM, Hoffmann J, Andraschko M, Werner J, Rittler P: Effects of malnutrition on compliction ates, lenght or hospital stay, and revenue in elective 8. Waitzberg DL, Caiaffa WT, Correia MI: Hospital malnutrition: the
Brazilian national survey (IBRANUTRI): a study of 4000 patients. Nutrition 2001, 17(7-8):573-580

9. Wyszynski DF, Perman M, Crivelli A: Prevalence of hospital 9. Wratutition in Argentina: preliminary results of a population-based (10. Nutrion 2003, 19(2):115-119.

0. Correia MI, Hegazi RA, Diaz-Pizarro Graf JI, Gomez-Morales G, Fuentes Gutierrez C, Goldin MF, et al. Addressing Disease-Related
Malnutrition in Healthcare: A Latin American Perspective. JPEN Parenter Enteral Nutr 2016, 40(3):319-325.

1. Studley HO: Percentage of weight loss: a basic indicator of surgical 16(4):141-3; discussion 140-1.
risk in patient with chon

12. Ho JW, Wu AH, Lee MW, Lau SY, Lam PS, Lau WS, et al. Malnutrition risk predicts surgical outcomes in patients undergoing 2015, 34(4):679-684.

13. Veterans Affairs Total Parenteral Nutrition Cooperative Study Group: Perioperative total parenteral nutrition in surgical patients. N wards $5 \mathrm{~A}$ and $5 \mathrm{~B}$ for their support throughout the stud Engl J Med 1991, 325(8):525-532
( parenteral nutrition for malnourished surgical patients. World J Surg

15. Zhong JX, Kang K, Shu XL: Effect of nutritional support on clinical outcomes in perioperative malnourished patients: a meta-analysis. Asia Pac J Clin Nutr 2015, 24(3):367-378.

16. Soeters PB, Schols AM: Advances in understanding and assessing 7. 17. Jensen GL, Mirtallo J, Compher C, Dhaliwal R, Forbes A, Grijalba for etiology-based diagnosis in the clinical practice setting from the International Consensus Guideline Committee. JPEN J Parenter Enteral Nutr 2010, 34(2):156-159.

18. Long CL, Schaffel N, Geiger JW, Schiller WR, Blakemore WS: Metabolic response to injury and illness: estimation of energy and protein needs from indirect calorimetry an.
Parenter Enteral Nutr 1979, 3(6):452-456.

19. Finnerty CC, Mabvuure NT, Ali A, Kozar RA, Herndon DN: The surgically induced stress response. JPEN J Parenter Enteral Nutr 2013,
$37(5$ Suppl):21S-9S.

20. Weimann A, Braga M, Harsanyi L, Laviano A, Ljungqvist O, Soeters P, et al. ESPEN Guidelines on Enteral Nutrition: Surgery including

21. Abunnaja S, Cuviello A, Sanchez JA: Enteral and parenteral nutrition in the perioperative period: state of the art. Nutrients 2013

22. Braga M: The 2015 ESPEN Arvid Wretlind lecture. Evolving concepts on perioperative metabolism and support. Clin Nutr 2016,

23. Andersen HK, Lewis SJ, Thomas S: Early enteral nutrition within 24h of colorectal surgery versus later commencement of feeding for postoperative complications. Cochrane Database Syst Rev 2006,

24. Braga M, Ljungqvist O, Soeters P, Fearon K, Weimann A, Bozzetti F, et al. ESPEN Guidelines on Parenteral Nutrition: surgery. Clin Nutr 2009, 28(4):378-386

25. Meier R, Ockenga J, Pertkiewicz M, Pap A, Milinic N, Macfie J, et al. ESPEN Guidelines on Enteral Nutrition: Pancreas. Clin Nutr 2006, 25(2):275-284

26. Gianotti L, Meier R, Lobo DN, Bassi C, Dejong CH, Ockenga J, et al. ESPEN Guidel 2809 , 2 : $428-435$,

27. Taylor BE, McClave SA, Martindale RG, Warren MM, Johnson DR, Braunschweig C, et al.Guidelines for the Provision and Assessment of
Nutrition Support Therapy in the Adult Critically Ill Patient: Society of Critical Care Medicine (SCCM) and American Society for Parentera and Enteral Nutrition (A.S.P.E.N.). Crit Care Med 2016, 44(2):390-438. 28. Takele Y, Adem E, Getahun M, Tajebe F, Kiflie A, Hailu A, R et al.Malnutrition in Healthy Individuals Results in Increased Mixed Cytokine Profiles, Altered Neutrophil Subsets and Function. PLoS One
2016, 11(8):e0157919. 29. van Bokhorst-de van der Schueren,M.A., Guaitoli PR, Jansma EP,

\section{$3(1): 39-58$.}

30. da Silva Fink J, Daniel de Mello P, Daniel de Mello E: Subjective literature. Clin Nutr 2015, 34(5):785-792.

1. Zohra Lukmanji AND Ellen Hertzmark Nicolas Mlingi, Vincent Assey, Godwin Ndossi, Wafaie Fawzi: Tanzania Food Composition Muhimbili University of of Pubic Health (HSPH), Boston, USA es Salaam - Tuzania and Tanzania Food and Nutrition Centre (TFNC), Dar ss Salaam - Tanzania and Harvard School of Pubic Health (HSPH), Boston, USA; 2008.

32. Government of Malawi: 2016/2017 FOOD INSECURITY RESPONSE PLAN. 2016,

3. Asiimwe SB, Muzoora C, Wilson LA, Moore CC: Bedside measures of malnutrition and association
Clin Nutr 2015, 34(2):252-256. Larouze B: Nutritional (impact of HIV infection). Eur J Clin Nutr 1999, 53(7):579-582.

35. Shah NR, Braverman ER: Measuring adiposity in patients: the utility of body mass index (BMI), percent body fat, and leptin. PLoS One 2012, 7(4):e33308.

36. Li H, Yang G, Xiang YB, Zhang X, Zheng W, Gao YT, Shu XO Body weight, fat distribution and colorectal cancer risk: a report from 2013, 37(6):783-789.

37. Prospective Studies Collaboration, Whitlock G, Lewington $S$, Sherliker P, Clarke R, Emberson J, et al. Body-mass index and cause-

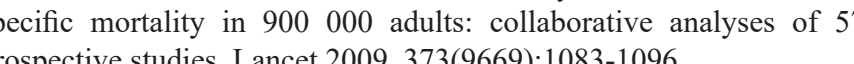
.

8. Abe Vicente M, Barao K, Silva TD, Forones NM: What are the most effective methods for assessment of nutritional status in outpatient 39. Ozbilgin S, Hanc V, Omur D, Ozbilgin M, Tosun M, Yurtlu S, et al. Morbidity and mortality predictivity of nutritional assessment tools in
the postoperative care unit. Medicine (Baltimore) 2016, 95(40):e5038. 40. Kondrup J, Allison SP, Elia M, Vellas B, Plauth M, Educational and Clinical Practice Con screening 2002. Clin Nutr 2003, 22(4):415-421,

41. Guerra RS, Fonseca I, Pichel F, Restivo MT, Amaral TF: Handgrip strength and associated factors in hospitalized patients. JPEN J Parenter Enteral Nutr 2015, 39(3):322-330.

42. Kotler DP, Tierney AR, Wang J, Pierson RN,Jr: Magnitude of bodyJ Clin Nutr 1989, 50(3):444-447.

43. Demling RH: Nutrition, anabolism, and the wound healing process: an overview. Eplasty 2009, 9:e9.
34. Niyongabo T, Henzel D, Ndayishimyie JM, Melchior JC, 